\title{
Characteristics of Arthrobacter Grown in Continuous Culture
}

\author{
By B. M. LUSCOMBE* AND T. R. G. GRAY \\ Hartley Botanical Laboratories, University of Liverpool, P.O. Box 147, Liverpool L69 $3 B X$
}

(Received 29 August 1973; revised 12 December 1973)

\begin{abstract}
SUMMARY
When Arthrobacter globiformis, A. simplex, A. crystallopoietes and A. atrocyaneus were grown in a chemostat, their morphology was related to growth rate. A transition from rod to coccus occurred at growth rates characteristic for each species; the higher the rate at which this transition occurred, the higher was the maximum specific growth rate. Organism size, concentration and viability were determined for $A$. globiformis at a range of dilution rates between $0.0 \mathrm{I} \mathrm{h}^{-1}$ and washout. Yield remained constant except at low dilution rates, although the number of organisms varied because of the changes in their size and shape. The percentage of viable organisms in the chemostat remained almost constant at dilution rates above $0.1 h^{-1}(92 \%)$ and was only slightly lowered even at dilution rates as low as $0.01 \mathrm{~h} \mathrm{~h}^{-1}$.

Rods grown in the chemostat and then placed in nutrient-free solutions divided and produced cocci which survived for about 56 days. Cocci survived for about 70 days. The respiration rate of freshly harvested rods was higher than that of cocci but within two days both fell to low levels.
\end{abstract}

\section{INTRODUCTION}

Arthrobacters are pleomorphic bacteria occurring as rods or cocci under different conditions. In batch culture, with appropriate media, they are rod shaped only during the exponential phase of growth, reverting to cocci in the stationary phase. Luscombe \& Gray (197I) found that when A. globiformis was grown in continuous flow culture the morphology was related to growth rate. A transition occurred within a narrow range of growth rates, above which organisms occurred as rods and below which they grew as cocci. The transition point was temperature dependent. Although changes in size have been reported for other bacteria grown in chemostats at different growth rates (Herbert, 1959), these took place gradually over a wide range of dilution rates, rather than sharply. The aim of the present work was to study this transition in greater detail in $A$. globiformis and to look for it in a range of Arthrobacter species. In addition, rods and cocci were grown in the same growth medium and their survival compared in nutrient-free conditions, which may have some relevance to the growth and survival of these bacteria in their natural soil environment.

\section{METHODS}

Growth of arthrobacters. Four species of Arthrobacter were grown in a chemostat with a working volume of $150 \mathrm{ml}$ and vortex aeration (Luscombe \& Gray, 197I). A. globiformis (NCIB10683), A. atrocyaneus (ICPB2396). A. simplex (AC4, J. Antheunisse, Wageningen) and $A$. crystallopoietes (NCIB9499) were grown at $25^{\circ} \mathrm{C}$ in the simple salts base $\mathrm{E}$ used by

\footnotetext{
* Present address: Department of Biochemistry and Microbiology, Rutgers University, New Brunswick, New Jersey, U.S.A.
} 
Owens \& Keddie (1969) with added biotin (I $\mu \mathrm{g} / \mathrm{l}$ ) and a limiting carbon source. Glucose $(0.2 \mathrm{~g} / \mathrm{l})$ was used for the growth of $A$. globiformis, $A$. atrocyaneus and $A$. simplex, while sodium succinate $(0.5 \mathrm{~g} / \mathrm{l})$ was used for $A$. crystallopoietes. Yeast extract $(2 \mathrm{mg} / \mathrm{l})$ was added to the medium for $A$. simplex. Low glucose concentrations were used to simulate the provision of small amounts of carbon in soil and to give populations where lack of oxygen or the development of acidic $\mathrm{pH}$ would not limit growth. The $\mathrm{pH}$ of the growth medium following stabilization of the chemostat was 6.75. The bacteria chosen represent strains common in the soil (Lowe \& Gray, 1972) and ones used by other laboratories examining the growth of arthrobacters in culture.

A fermentation vessel with a working volume of $2725 \mathrm{ml}$ (Biotec) was used to grow the larger quantities of $A$. globiformis required for studies on endogenous respiration and survival. A glucose concentration of $0.6 \mathrm{~g} / \mathrm{l}$ was used and dilution rates of $0.05 \mathrm{~h}^{-1}$ and $0.3 \mathrm{~h}^{-1}$ established to produce cocci and rods respectively. Higher glucose concentrations were used in this chemostat since oxygen could be kept at a higher level and $\mathrm{pH}$ could be controlled.

Organism size. A drop of $0.033 \mathrm{M}$-phosphate buffer was placed on the air-dried smear of bacteria and their radii measured at $\times 1600$ magnification using a phase-contrast microscope. Volumes were calculated from these data.

Organism yield. Organisms were counted, using a Helber counting chamber (Hawksley Ltd, London). Dry weights were determined by centrifuging suspensions, washing them and drying to a constant weight at $110{ }^{\circ} \mathrm{C}$.

Harvesting. Bacteria were harvested, washed three times in $0.033 \mathrm{M}$-phosphate buffer by centrifugation at $3000 \mathrm{~g}$ for $15 \mathrm{~min}$, and suspended in further buffer at $5 \times 10^{9} / \mathrm{ml}$. This suspension was used for studies on endogenous respiration and survival in nutrient-free conditions.

Viability. This was determined by a modification of the slide culture technique of Postgate, Crumpton \& Hunter (I96I) using a medium of yeast extract (Difco) $0.7 \%$, glucose $1.0 \%$ and ionagar (Oxoid) $\mathrm{I} \cdot 2 \%$. Blocks $(5 \times 5 \mathrm{~mm})$ were cut from a layer of agar 0.75 to $\mathrm{I} \cdot 0 \mathrm{~mm}$ deep and inoculated with organisms from the chemostat to give approximately 30 bacteria/field of view. After the inoculum had dried, a coverslip was placed on the agar block which was put in a moist chamber for incubation. Cultures were incubated for up to $16 \mathrm{~h}$, when colonies of 8 to 16 bacteria had formed from viable organisms and dead forms remained visible as single bacteria. Slide cultures were examined using phasecontrast microscopy $(\times 640)$, and by counting the number of colonies and dead bacteria the percentage viability could be determined.

Endogenous respiration. This was measured as the rate of oxygen uptake of bacterial suspensions in a Gilson differential respirometer (Gilson Medical Electronics, France) and using a polarographic electrode (Rank Bros, Cambridge).

Maximum specific growth rate. This was determined by the washout technique of Jannasch (1969). Bacteria were grown in the chemostat at a dilution rate of $0.35^{\mathrm{h}^{-1}}$ until a steady state had been reached and then the dilution rate was increased to $0.43 \mathrm{~h}^{-1}$ for $4 \mathrm{~h}$. Numbers in the culture were determined at this time and after a further 2, 4, I6, I8, 20, 22 and $24 \mathrm{~h}$ during washout. Tempest (1970) has shown that

$$
\ln x_{1}-\ln x_{2}=\left(D-\mu_{m}\right)\left(t_{2}-t_{1}\right),
$$

where $x_{1}$ and $x_{2}$ are organism concentrations at times $t_{1}$ and $t_{2}$ respectively, $D$ is the dilution rate and $\mu_{m}$ the maximum specific growth rate. The slope in a plot of $\ln x$ against time is therefore equal to $D-\mu_{m}$. 


\section{RESULTS}

\section{Morphogenesis in Arthrobacter species}

All four Arthrobacter species investigated underwent a transformation from coccus to rod form. A. simplex became rod-shaped at dilution rates above $0.05 \mathrm{~h}^{-1}, A$. crystallopoietes at dilution rates above $0.15 \mathrm{~h}^{-1}$ and $A$. atrocyaneus and $A$. globiformis at dilution rates above $0.25 \mathrm{~h}^{-1}$. The dilution rate at which the transition took place appeared to be a function of the maximum specific growth rate of the organism, since the higher the value for $\mu_{m}$ the higher the dilution rate at which the change occurred. $A$. simplex washed out of the chemostat at dilution rates above $0 . \mathrm{I} \mathrm{h}^{-1}, A$. crystallopoietes at rates above $0.25 \mathrm{~h}^{-1}$, A. globiformis at rates above $0.35 \mathrm{~h}^{-1}$, and $A$. atrocyaneus at rates above $0.4 \mathrm{~h}^{-1}$. The relationship of the transition point to maximum specific growth rate was also found by Luscombe \& Gray (197I) when they studied the effect of temperature on this phenomenon. The highest values for $\mu_{m}$ occurred between 25 and $30^{\circ} \mathrm{C}$, and the transition also occurred at the highest dilution rates in this temperature range. The pigmentation of $A$. globiformis also changed markedly with growth rate as the rods produced an indigoidine-type pigment and a carotenoid, while the cocci produced only a carotenoid. Cultures of rods were therefore blue and cocci yellow.

Increases in mean volume and shape (plotted as the ratio of the shorter axis to the longer axis) of A. globiformis occurred simultaneously within a limited range of dilution rates between 0.2 and $0.3 \mathrm{~h}^{-1}$ (Fig. I). The increase in size was due to an increase in the length of one axis only. The distributions of individual bacterial volumes at a number of steady states were skewed (Fig. 2); this agrees with the observation by Powell (1956) of a J-shaped distribution of organism ages, most of the organisms in the culture being small and presumably young.

\section{Variation of yield with dilution rate}

The yield of $A$. globiformis at different dilution rates is shown both as numbers and dry weights. Numbers of organisms in steady state cultures varied at different dilution rates, reaching the highest values between 0.1 and $0.15 \mathrm{~h}^{-1}$ (Fig. 3). At dilution rates between 0.3 and $0.35 \mathrm{~h}^{-1}$ the numbers remained constant, but they fell rapidly at higher dilution rates as the culture became unstable. A semi-logarithmic plot of the rate of washout of $A$. globiformis from the chemostat at a dilution rate of $0.43 \mathrm{~h}^{-1}$ (Fig. 4) had a slope of 0.06 , and so $\mu_{m}$ must be $0.37 \mathrm{~h}^{-1}$. This value is higher than the highest steady state dilution rate that can be achieved because some of the bacteria in the chemostat are non-viable (see below).

Chemostat theory predicts that organism concentration should remain constant unless the dilution rate is so low that the maintenance energy requirement becomes a relatively important part of the energy used (Veldkamp, 1968). However, this would only be true if organism size were constant over the whole range of dilution rates, which is not the case with Arthrobacter. Nevertheless, the yield measured in terms of dry weight should still be constant in these organisms and this proved to be so (Fig. 3). The decrease in the dry weight yield at dilution rates below $0 \cdot 1 \mathbf{h}^{-1}$ was less than that reported for other organisms, e.g. Aerobacter aerogenes (Herbert, 1959), suggesting that the energy required for maintenance of Arthrobacter is also smaller. Chapman (personal communication) has now determined the maintenance coefficient for $A$. globiformis NCIBIO683 as $0.0088 \mathrm{~h}^{-1}$, confirming this view. 


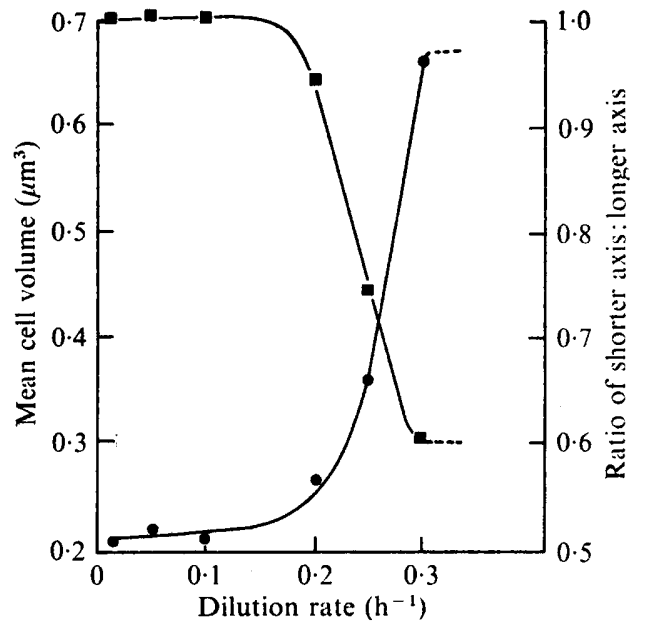

Fig. I
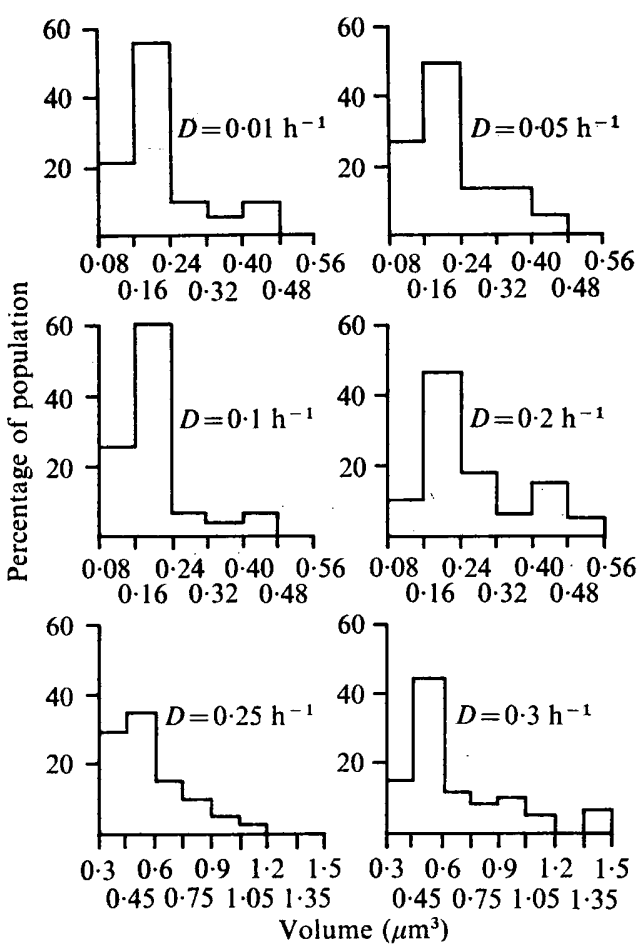

Fig. 2

Fig. I. Effect of dilution rate on the mean volume and shape of A. globiformis NCIBIO683. $\boldsymbol{D}$, Shape (ratio of the lengths of the shorter axis and longer axis); $\bullet$, volume in $\mu \mathrm{m}^{3}$. For details of growth conditions see text.

Fig. 2. Distribution of individual rod and coccus volumes of $A$. globiformis NCIBIO683 grown at different dilution rates in a chemostat. For details see text.

\section{Variation of viability with dilution rate}

The percentage of viable organisms in the chemostat cultures was found to be almost constant at dilution rates above $0 . \mathrm{I} \mathrm{h}^{-1}(92 \%)$ and was only slightly reduced even at dilution rates as low as $0.01 \mathbf{h}^{-1}$. A. globiformis differed markedly from Aerobacter aerogenes (Fig. 5). Postgate \& Hunter (1962) showed and later Tempest, Herbert \& Phipps (1967) confirmed, that although the viability of $A$. aerogenes was between 95 and $100 \%$ at dilution rates above $0.1 \mathrm{~h}^{-1}$, it dropped to about $65 \%$ at dilution rates of $0.0 \mathrm{I} \mathrm{h}^{-1}$.

Tempest (1970) pointed out that when non-viable organisms were present in a culture, the viable organisms must grow at a rate greater than the dilution rate since they have to replenish the viable and non-viable organisms lost in the effluent. He showed that

$$
\mu_{m}^{*}=\frac{D \ln 2}{\ln 2 \alpha},
$$

where $\mu_{m}^{*}$ is the maximum specific growth rate for the viable population and $\alpha$ is an index of viability, equal to $V+\frac{1}{2}$ where $V$ is the fraction of viable organisms in the culture.

In the case of $A$. globiformis, the highest steady state dilution rate was $0.35 \mathrm{~h}^{-1}$ and the viability index 0.96 . This gives a value for $\mu_{m}^{*}$ of $0.37 \mathrm{~h}^{-1}$, confirming the result obtained earlier by Jannasch's (1969) washout method. 


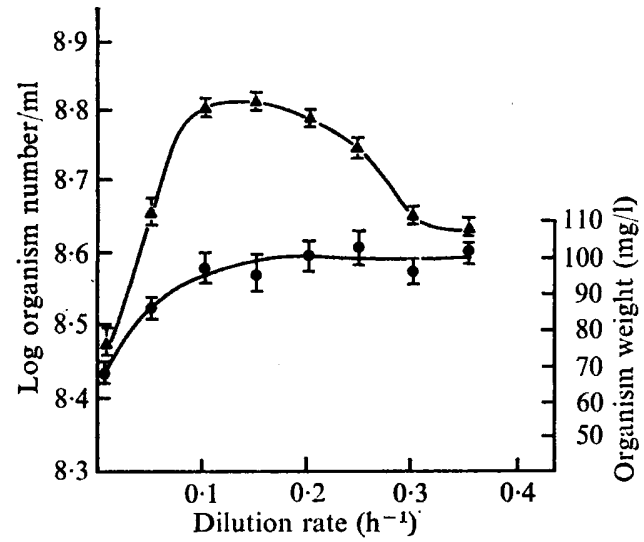

Fig. 3

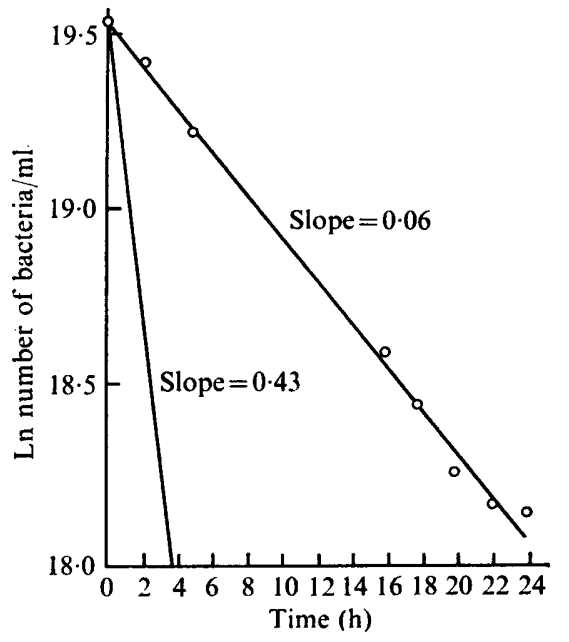

Fig. 4

Fig. 3. Effect of dilution rate on yield of A. globiformis NCIBIO683 in terms of weight and concentration. $\Delta$, Number of organisms $/ \mathrm{ml}$; $O$, weight of organisms (mg/l). For details see text.

Fig. 4. Rate of washout of $A$. globiformis NCIBI0683 from a chemostat $\left(D=0.43 \mathrm{~h}^{-1}\right)$. $O$, Rate of washout of organisms; —_, predicted rate of washout in the absence of growth.

\section{Rate of endogenous respiration}

Suspensions of organisms in $0.033 \mathrm{M}$-phosphate buffer were incubated at $25^{\circ} \mathrm{C}$ on a rotary shaker ( $150 \mathrm{rev} . / \mathrm{min}$ ) and sampled after $0,10,24$ and $48 \mathrm{~h}$ and 7 days to determine the rate of oxygen uptake. Higher values of $Q_{\mathrm{o}_{2}}$ were obtained using oxygen polarography but, using either technique, the rates of oxygen uptake fell rapidly in both rods and cocci (Fig. 6). Gilson respirometry gave initial $Q_{\mathrm{O}_{2}}$ values of $\mathrm{I} \cdot 74$ for cocci and $7 \cdot 33$ for rods, but after 2 days these had both fallen to a relatively stable level of 0.45 . These values are very low compared with the value of 75 obtained by Herbert (1959) for Aerobacter aerogenes but are similar to the values 4 to 6 reported by Sobek, Charba \& Foust (1966) for Azotobacter agilis, another soil organism. A rapid drop in the rate of oxygen uptake during the early stages of starvation has also been reported by Mulder, Deinema, van Veen \& Zevenhuizen (1962) and Boylen \& Ensign (1970a) for Arthrobacter.

\section{Organism numbers and viability during starvation}

Measurements of numbers and viability were made after $0,7,14,28,42,56$ and 70 days in $0.033 \mathrm{M}$-phosphate buffer at $25^{\circ} \mathrm{C}$. The number of rods, expressed as a percentage of the original suspension, increased during the first 7 days but then declined (Fig. 7). The increase in numbers was accompanied by fragmentation of the rods to form cocci and after 70 days the suspension consisted almost entirely of cocci (Fig. 8). This might account for the convergence of the endogenous respiration rates of cocci and 'rods' observed after 2 to 7 days in the Gilson respirometer. The rate of reduction of total numbers in the suspension was slightly greater for rods than for cocci, despite the fragmentation process. There was a $30 \%$ reduction in the number of cocci and a $40 \%$ reduction in the number of 'rods' (based on the number of organisms present after 7 days) over the 70 day incubation period. 


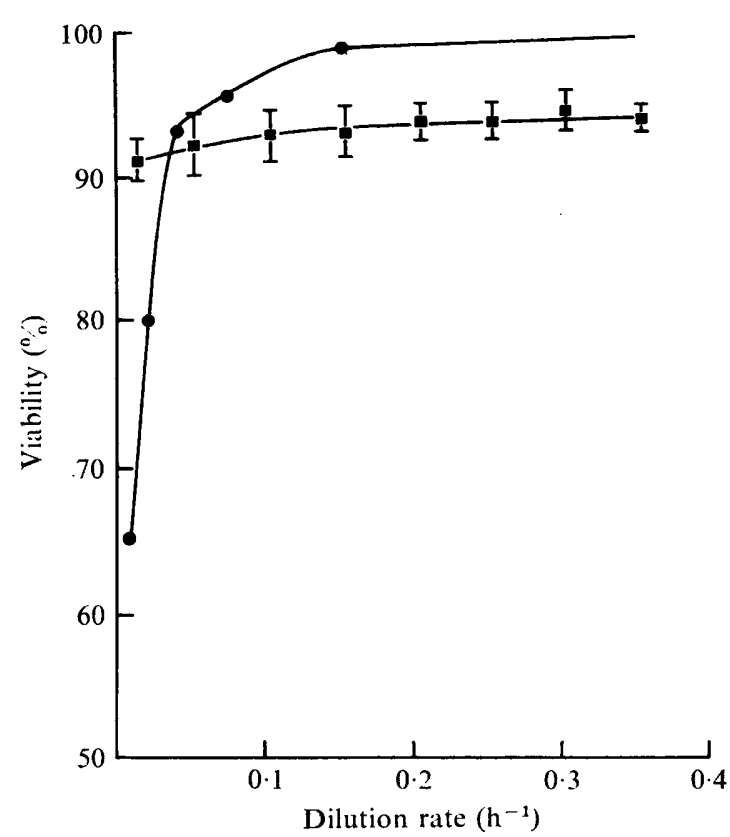

Fig. 5

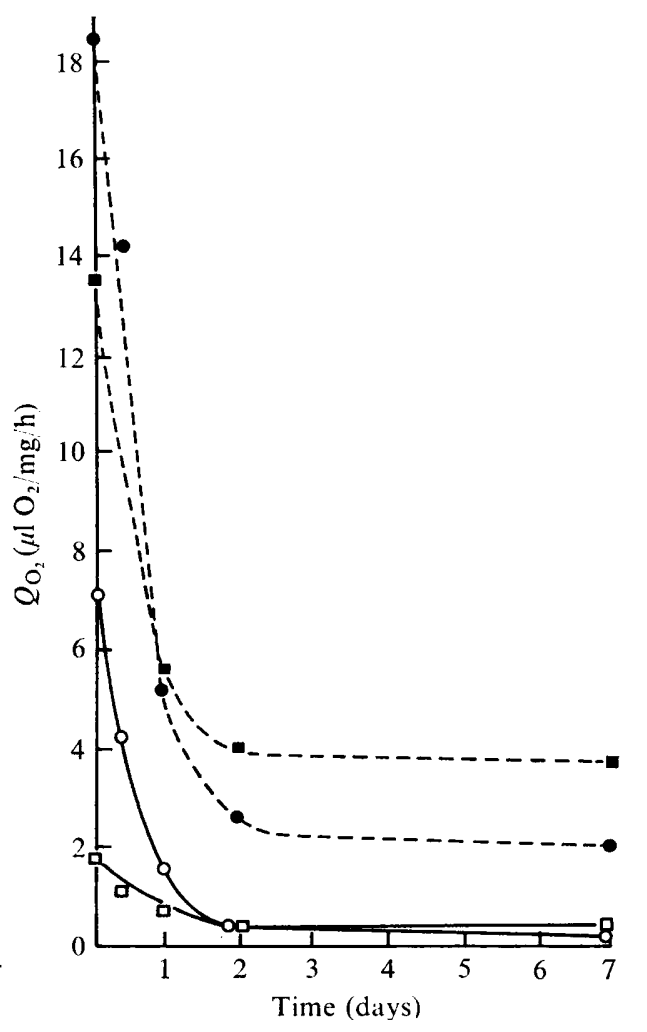

Fig. 6

Fig. 5. Steady state viabilities of A. globiformis NCIB 10683 compared with Aerobacter aerogenes (data of Tempest et al. 1967) under carbon-limiting conditions in a chemostat. $\square, A$. globiformis NCIBI0683; , A. aerogenes. For details see text.

Fig. 6. Oxygen uptake by $A$. globiformis NCIBI0683 after pre-incubation for different times in 0.033 M-phosphate buffer $(\mathrm{pH} 7 \cdot 0)$ at $25^{\circ} \mathrm{C}$, measured by respirometry $(O$, rods; $\square$, cocci) and by polarography $\left(\boldsymbol{O}\right.$, rods; $\square$, cocci). For details see text. $Q_{\mathbf{o}_{2}}$ values were corrected by assuming that only 'viable' organisms respire.

The viability of the 'rods' fell at a faster rate than that of cocci and no viable organisms were observed at the 70-day sampling time (Fig. 9). Presumably, some energy is used in the production of wall material during fragmentation of the rods and so less is available for the maintenance of the organisms. The initial increase in total numbers as rods became cocci was smaller than might be expected if each rod gave rise to at least two cocci. It must be assumed, therefore, that on formation one of each pair of cocci lysed or that rods which did not form cocci lysed.

\section{DISCUSSION}

Two theories have been put forward to explain the change of cocci to rods in arthrobacters. Krulwich \& Ensign (1969) concluded that certain chemicals, e.g. succinate, were required to induce rod formation in $A$. crystallopoietes and that in the absence of inducers, rods would revert to cocci. Luscombe \& Gray (197I) suggested that $A$. globiformis morphology was related to growth rate. The studies reported here have shown that growth rate was a determining factor of morphology in a range of arthrobacters, including $A$. crystallo- 


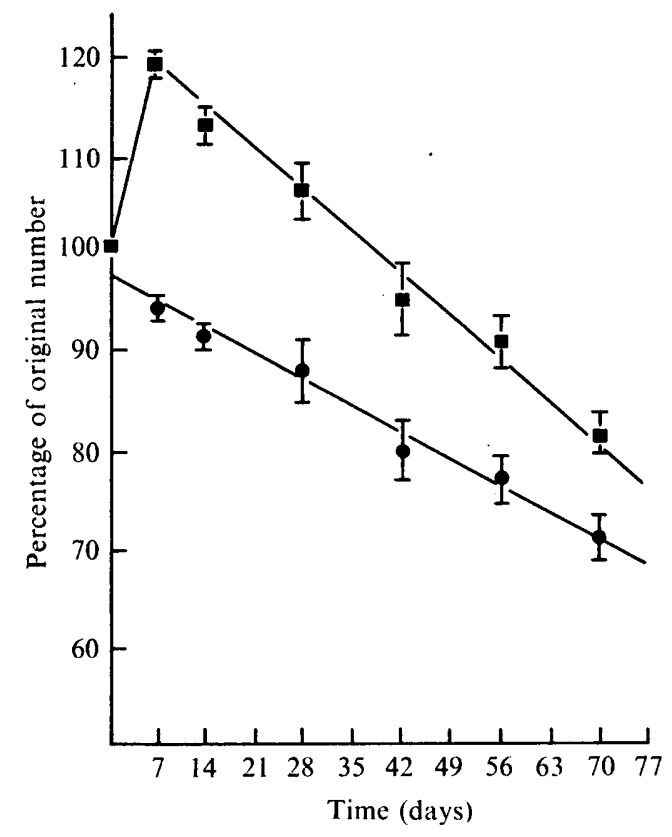

Fig. 7. Changes in total numbers of $A$. globiformis NCIBI0683 during incubation at $25^{\circ} \mathrm{C}$ in 0.033 M-phosphate buffer (pH 7.0). $\mathbf{D}$, Rods; $\bullet$, cocci.

poietes growing on succinate. In the measurement of organism size, the drying of bacteria in films may have caused some shrinkage but it was considered that this would be relatively uniform. Boylen \& Pate (1973) suggested that arthrobacters form two physiologically distinct types of cocci: those formed during exponential growth and those existing in the stationary phase. However, it seems more likely that the cocci found in stationary phase cultures were not distinct types of organism but merely older organisms formed when the rate of growth of the culture was declining.

The loss of viability of bacteria in chemostat cultures at low growth rates led Tempest, Herbert \& Phipps (1967) to suggest that bacteria have a 'minimum growth rate'. At growth rates below this, a proportion of the culture becomes non-viable and the remainder continues to grow at the minimum rate. In reviewing evidence for this, Pirt (1972) suggested that below a growth rate equivalent to $0.06 \mu_{m}$ all or part of a population shows a loss of viability. However, A. globiformis showed little loss of viability at growth rates of $0.0 \mathrm{I} \mathrm{h}^{-1}$ which is $0.027 \mu_{m}$, suggesting that if a minimum growth rate exists in Arthrobacter it is lower than was previously suggested for Aerobacter and Escherichia.

Bacteria grown under carbon-limiting conditions survived even in the absence of nutrients for remarkably long periods. Boylen \& Ensign (I970a) found that A. crystallopoietes grown in batch culture survived for similar periods of time. It is not clear whether the long survival times are related to the presence of storage materials and their low rate of utilization. Boylen \& Ensign (1970 b) found much storage material even after long starvation, but although cocci contained more storage material than rods they survived for similar times. They suggested that rods used up their reserve materials more quickly and, in the present investigation, this may be because of the energy requirement for division of rods in nutrientfree buffer. However, Boylen \& Ensign (1970 $b$ ) found that their rods did not fragment and produce cocci during starvation. 

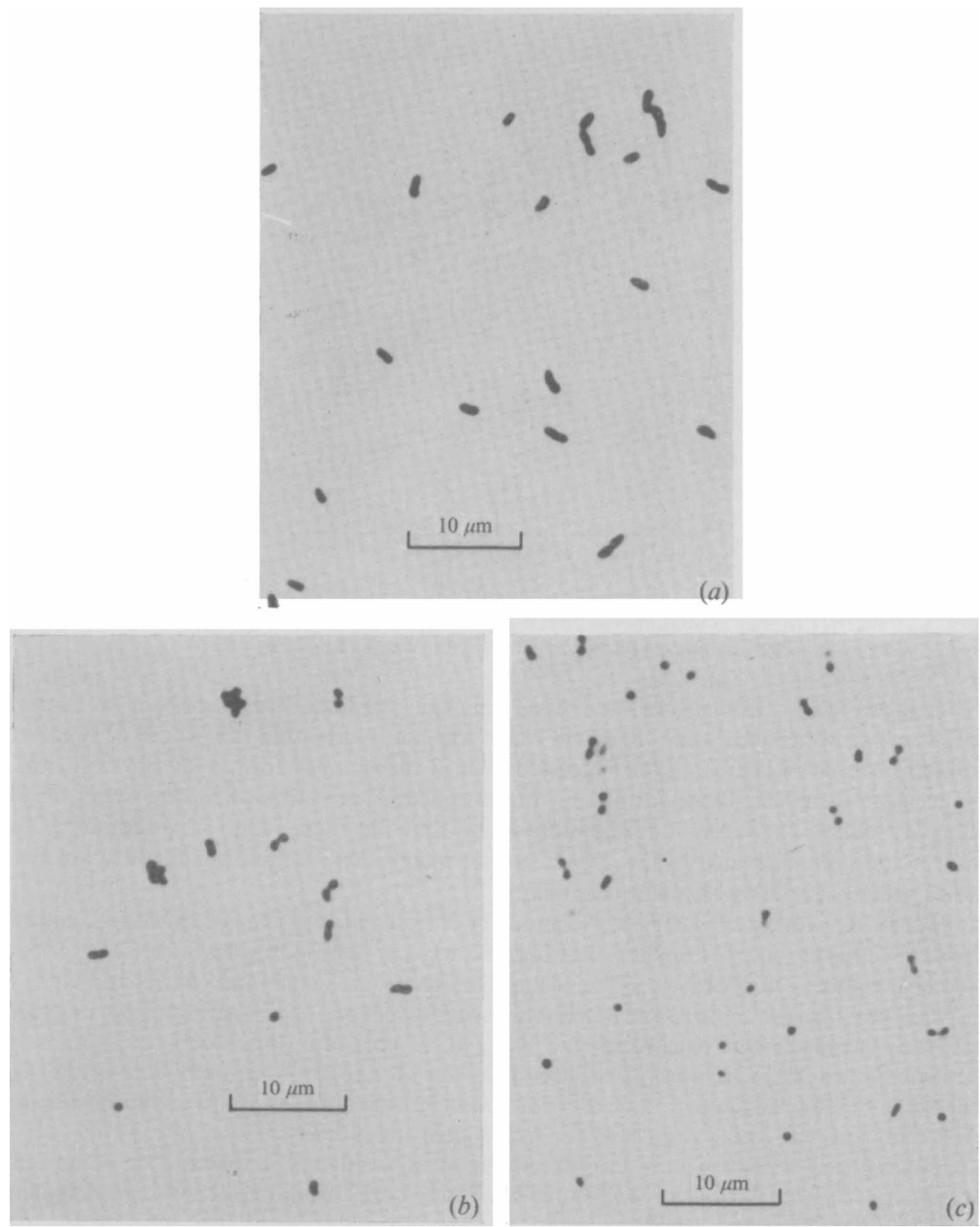

Fig. 8. A. globiformis NCIBIO683 harvested from the chemostat $\left(D=0.3 \mathrm{~h}^{-1}\right)$ and examined either at once $(a)$ or after incubation at $25^{\circ} \mathrm{C}$ in $0.033 \mathrm{M}$-phosphate buffer for 7 days $(b)$ or for 70 days $(c)$. 


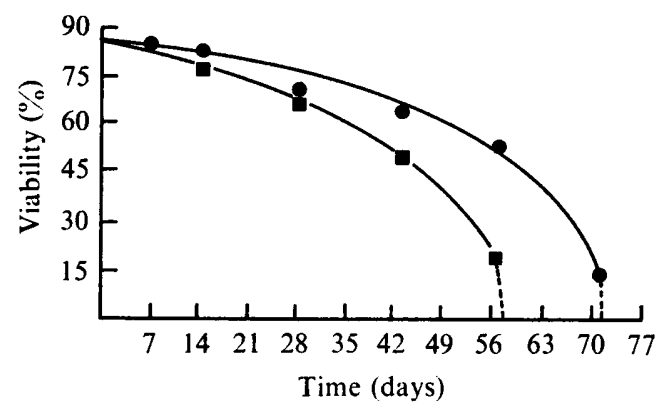

Fig. 9. Loss of viability of $A$. globiformis NCIBI0683 during incubation at $25{ }^{\circ} \mathrm{C}$ in $0.033 \mathrm{M}$-phosphate buffer (pH 7.0). $\mathrm{D}$, Rods; 0 , cocci.

These properties are presumably connected with the comparative success of arthrobacters in environments like soil where the ability to retain viability at low growth rates or during resting periods is important. In soil there may be other environmental conditions which are unfavourable, e.g. water availability, $\mathrm{pH}$, etc. The effect of such factors on $A$. globiformis is now being investigated. Boylen \& Ensign (1970a) expressed surprise that cocci did not survive longer than rods during starvation. However, the rods and cocci for those experiments were produced in different media and so their survival times are not strictly comparable. We have found that rods of $A$. globiformis do lose their viability a little faster when they are grown in the same medium as cocci. However, this is unlikely to be important to bacteria in natural environments since rods are probably only rarely produced. Gray \& Williams (I97I) have shown that the generation times of bacteria in soil are not likely to be less than a day, except for very short periods of time, and so most arthrobacters would grow as cocci.

We wish to thank the Science Research Council for grants and Dr J. Hopton, University of Birmingham, for his advice on the design of the chemostat.

\section{REFERENCES}

BOYLEN, C. W. \& ENSIGN, J. C. (I970a). Long term starvation survival of rod and spherical cells of Arthrobacter crystallopoietes. Journal of Bacteriology 103, 569-577.

Boxlen, C. W. \& Ensign, J. C. (1970 b). Intracellular substrates for endogenous metabolism during longterm starvation of rod and spherical stage cells of Arthrobacter crystallopoietes. Journal of Bacteriology 103, 578-587.

Boylen, C. W. \& Pate, J. L. (1973). Fine structure of Arthrobacter crystallopoietes during long term starvation of rod and spherical stage cells. Canadian Journal of Microbiology 19, 1-5.

Gray, T. R. G. \& Williams, S. T. (197I). Microbial productivity in soil. In Microbes and Biological Productivity, Symposia of the Society for General Microbiology 21, 256-286.

Herbert, D. (1959). Some principles of continuous culture. In Recent Progress in Microbiology, pp. $381-396$. Edited by G. Tunevall. Oxford: Blackwell's Scientific Publications.

JANNASCH, H. W. (1969). Estimations of bacterial growth rates in natural water. Journal of Bacteriology 99 , I56-I60.

KRULWICH, T. A. \& ENSIGN, J. C. (I969). Alteration of glucose metabolism of Arthrobacter crystallopoietes by compounds which induce sphere-rod morphogenesis. Journal of Bacteriology 97, 526-534.

Lowe, W. E. \& GrAY, T. R. G. (1972). Ecological studies on coccoid bacteria in a pine forest soil. I. Classification. Soil Biology and Biochemistry 4, 459-467.

LusCombe, B. M. \& GRAY, T. R. G. (1971). Effect of varying growth rate on the morphology of Arthrobacter. Journal of General Microbiology 69, 433-434. 
Mulder, E. G., Deinema, M. H., van Veen, W. L. \& Zevenhuizen, L. P. T. M. (I962). Polysaccharides, lipids and poly- $\beta$-hydroxybutyrate in micro-organisms. Recueil des travaux chimiques des Pays-Bas et de la Belgique 8r, 797-809.

OWENS, J. D. \& KEDDIE, R. M. (1969). The nitrogen nutrition of soil and herbage coryneform bacteria. Journal of Applied Bacteriology 32, 338-347.

PIRT, S. J. (1972). Prospects and problems in continuous flow culture of micro-organisms. In Environmental Control of Cell Synthesis and Function, pp. 55-64. Edited by A. C. R. Dean, S. J. Pirt \& D. W. Tempest. London: Academic Press.

Postgate, J. R., Crumpton, J. E. \& Hunter, J. R. (196I). The measurement of bacterial viabilities by slide culture. Journal of General Microbiology 24, 15-24.

Postgate, J. R. \& Hunter, J. R. (1962). The survival of starved bacteria. Journal of General Microbiology 29, $233-263$.

Powell, E. O. (1956). Growth rate and generation time of bacteria with special reference to continuous culture. Journal of Microbiology 15, 492-51 I.

Sobek, J. M., Charba, J. F. \& Foust, W. N. (1966). Endogenous metabolism of Azotobacter agilis. Journal of Bacteriology 92, 687-695.

TEMPEST, D. W. (1970). The continuous culture of micro-organisms: I. Theory of the chemostat. In Methods in Microbiology, vol. 2, pp. 259-276. Edited by J. R. Norris \& D. W. Ribbons. London: Academic Press.

Tempest, D. W., Herbert, D. \& PhIPps, P. J. (1967). Studies on the growth of Aerobacter aerogenes at low dilution rates in a chemostat. In Microbial Physiology and Continuous Culture, pp. 240-253. Edited by E. O. Powell, C. G. T. Evans, R. E. Strange \& D. W. Tempest. London: H.M.S.O.

VELDKAMP, H. (1968). Bacterial physiology. In The Ecology of Soil Bacteria, pp. 20I-219. Edited by T. R. G. Gray \& D. Parkinson. Liverpool: Liverpool University Press. 